structure (or, equivalently, by a commutative algebra of observables). The essential difference between classical systems and quantum systems is not the occurrence of Planck's constant of action (classical systems may very well depend on Planck's constant) but the fact that quantum systems are entangled by Einstein-Podolsky-Rosen correlations. This holistic feature of quantum mechanics may be difficult to grasp for a scientist educated in the classical Cartesian tradition. Thirteen well-selected papers of this collection document the experimental reality of the holistic non-separability of quantum systems.

In order to understand measurements, we must understand the existence of classical subsystems in a quantum world. That is the main problem and a tough one. If it is solved, a measurement can be described by a channel (in the sense of Umegaki) which transfers the state of the quantum object to a state of the classical output system. Much research in this field has been done in the past decades and many new important results are known. None of them is mentioned in the book. Equally disappointing are the commentaries. For example, in their "Guide to Some Further Literature" the editors say that Gleason's theorem "simplified the axiomatics of quantum mechanics". Such a phrasing is grossly misleading: Gleason's theorem is a conceptually important result explaining why linear functionals can be used to

\section{Mechanics of physics}

\section{Abraham Pais}

The Quantum World.

By J.C. Polkinghorne.

Longman: 1984. Pp.100. Hbk

£7.95, \$14.95.

THE Curate of St Michael and All Angels in Bedminster is an interesting man. He knows that "science has achieved its success by restricting itself to a certain type of inquiry", and he was elected FRS for contributing to this success. He knows too that there are other issues which are essential for a grasp of our World but which are beyond the ken of science. "God is not to be put to the test."

These sayings of the Curate, culled from his recent article in the London Times, made me turn to his new book, The Quantum World, with the confidence that it would not serve up that blend of the material and the mystic which of late seems so palatable to so many. I was not disappointed. This book is short and lucid. It demands thinking but no knowledge of higher mathematics. Its language is simple. Having been reared on the principle that it is not nice to use eight-letter words in public, I am happy to report that the word ontology occurs only once. Formulae are describe quantum states. Another example of the editors' disregard of present research is to do with the irreversible behaviour of some quantum systems. The reprinted papers deal with ergodic properties and are certainly of historic interest, but helpful comments are lacking. I think it should have been the editors' duty to inform the non-specialist why ergodic properties alone are irrelevant and why one has to require stronger mixing properties.

In an anthology of this sort, some arbitrary decisions are unavoidable. But still it is hard to understand why the editors decided not to reproduce the thoughtful and masterly "Quantum Physics and Philosophy, Causality and Complementarity" by Bohr (1958) and "On the Interpretation of Quantum Mechanics" by Fock (1957).

Although the many omissions may be regretted, this set of papers makes fascinating reading. The editors expect that their collection can be used as a source book for a seminar on the subject. The historical aspects are certainly well covered so it is, indeed, an obvious starting point for those entering the field. But it far from represents the state of the art, and no reader should suppose that the book will allow him to catch up with modern developments.

Hans Primas is Professor in the Laboratorium für Physikalische Chemie at ETH-Zentrum, Zürich.

given here and there but mainly to show what they look like. Nine brief appendices introduce the reader to more arcane subjects including a half-page derivation of John Bell's celebrated criterion for the validity of quantum mechanics which has recently been subjected to stringent laboratory tests. (No surprises: quantum mechanics works.) A helpful glossary is also included. Princes and Poppers alike will derive benefit from this handsomely produced volume.

"Professional practitioners of quantum mechanics can calculate away without worrying about the outcome of the debates about the foundations of their subject" (p.16) which centre on the question: is it necessary to include a specification of the experimental arrangement in defining what we mean by a physical phenomenon? "No", says the classical mechanic who uses the code words: objective reality. "Yes", says the quantum mechanic whose code word is complementarity and who bases his argument on the ineluctable indeterminacy principle. After giving a clear account of the transition from the classical to the quantum era, the author guides us through the quantum arguments by the fitting device of a tour through thought experiments which illuminate this subtle issue. One such experiment is that of Einstein, Podolsky and Rosen. I regret that the author follows others by referring to the
EPR result as a paradox. The EPR paper, logically impeccable, merely shows that objective reality and complementarity cannot both be true. Einstein never called it a paradox. Nor was he "wistful[ly] longing after a determinate hidden variable theory" (p.73). He never referred to "hidden variables" in his writings and in fact of ten called them a cheap way out.

In addressing these questions of principle, care with language is of the essence. I cannot subscribe to the author's statement (p.60): "Measurement involves an intervention by our everyday world into

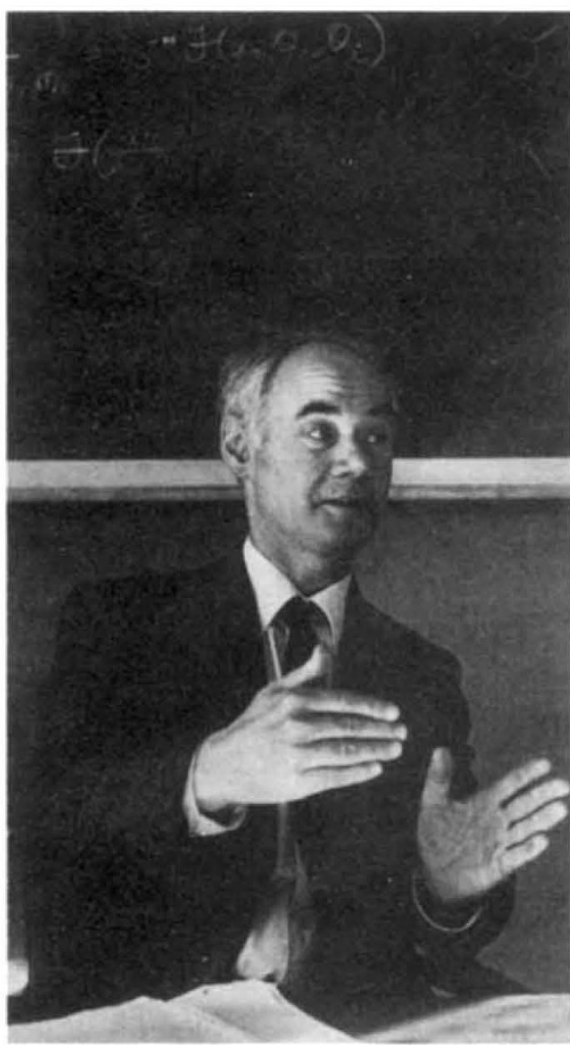

John Polkinghorne - "testimony to the fact that physics is necessary but not sufficient for salvation, that science and religion can coexist in harmonious complementarity".

the quantum world". I would rather say with Bohr: "There is no quantum world

It is wrong to think that the task of physics is to find out how nature is"' (p.79). May we then not ask what the World is? Of course we may, but here physics "restricting itself to a certain type of inquiry", cannot give answers. The author's life as well as his oeuvre, especially this lovely little book, bear testimony to the fact that physics is necessary but not sufficient for salvation, that science and religion can coexist in harmonious complementarity. He would agree, I think, with the quantum mechanic's reply to Einstein's assertion that God does not play dice: how do you know?

Abraham Pais is Professor of Physics at the Rockefeller University, New York. His biography of Albert Einstein. 'Subtle is the Lord first published in 1982, has recently been issued in paperback by Oxford University Press. 